Ref. SISSA 36/2010/EP

Ref. TUM-HEP $763 / 10$

Ref. IPPP/10/42, DCTP/10/84

\title{
TeV Scale See-Saw Mechanisms of Neutrino Mass Generation, the Majorana Nature of the Heavy Singlet Neutrinos and $(\beta \beta)_{0 \nu}$-Decay
}

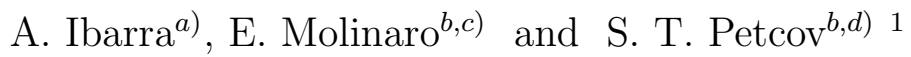 \\ a) Physik-Department T30d, Technische Universität München, \\ James-Franck-Straße, 85748 Garching, Germany. \\ b) SISSA and INFN-Sezione di Trieste, Via Bonomea 265, 34136 Trieste, Italy. \\ c) IPPP, Durham University, Durham DH1 3LE, UK. \\ ${ }^{d)}$ IPMU, University of Tokyo, Tokyo, Japan.
}

\begin{abstract}
It is shown that the Majorana nature of the heavy neutrinos $N_{j}$ having masses in the range of $M_{j} \sim(100-1000) \mathrm{GeV}$ and present in the TeV scale type $\mathrm{I}$ and inverse see-saw scenarios of neutrino mass generation, is unlikely to be observable in the currently operating and future planned accelerator experiments (including LHC) due to the existence of very strong constraints on the parameters and couplings responsible for the corresponding $|\Delta L|=2$ processes, $L$ being the total lepton charge. If the heavy Majorana neutrinos $N_{j}$ are observed and they are associated only with the type I or inverse see-saw mechanisms and no additional TeV scale "new physics", they will behave like Dirac fermions to a relatively high level of precision, being actually pseudo-Dirac particles. The observation of effects proving the Majorana nature of $N_{j}$ would imply that these heavy neutrinos have additional relatively strong couplings to the Standard Model particles (as, e.g. in the type III see-saw scenario), or that light neutrino masses compatible with the observations are generated by a mechanism other than see-saw (e.g., radiatively at one or two loop level) in which the heavy Majorana neutrinos $N_{j}$ are nevertheless involved.
\end{abstract}

\section{Introduction}

The experiments with solar, atmospheric, reactor and accelerator neutrinos [1, 2, 3, 4, 5, 6, 7, 8, 9, 10, 11, 12 have provided compelling evidences for the existence of flavour neutrino oscillations [13, 14] caused by nonzero neutrino masses and neutrino mixing. These data imply the presence of neutrino mixing in the weak charged lepton current:

$$
\nu_{l \mathrm{~L}}(x)=\sum_{j} U_{l j} \nu_{j \mathrm{~L}}(x), \quad l=e, \mu, \tau,
$$

where $\nu_{l L}$ are the flavour neutrino fields, $\nu_{j \mathrm{~L}}(x)$ is the left-handed (LH) component of the field of the neutrino $\nu_{j}$ possessing a mass $m_{j}$ and $U$ is a unitary matrix - the PontecorvoMaki-Nakagawa-Sakata (PMNS) neutrino mixing matrix [13, 14, 15].

\footnotetext{
${ }^{1}$ Also at: Institute of Nuclear Research and Nuclear Energy, Bulgarian Academy of Sciences, 1784 Sofia, Bulgaria
} 
All compelling neutrino oscillation data can be described assuming 3-flavour neutrino mixing in vacuum. The data on the invisible decay width of the $Z^{0}$-boson is compatible with only 3 light flavour neutrinos coupled to $Z^{0}$ (see, e.g. [16]). The number of massive neutrinos $\nu_{j}, n$, can, in general, be bigger than $3, n>3$, if, for instance, there exist righthanded $(\mathrm{RH})$ sterile neutrinos [15] and they mix with the LH flavour neutrinos. It follows from the existing data that at least 3 of the neutrinos $\nu_{j}$, say $\nu_{1}, \nu_{2}, \nu_{3}$, must be light, $m_{1,2,3} \lesssim 1 \mathrm{eV}$, and must have different masses, $m_{1} \neq m_{2} \neq m_{3}$. At present there are no compelling experimental evidences for the existence of more than 3 light neutrinos.

As is also well known, the data on the absolute scale of neutrino masses (including the data from ${ }^{3} \mathrm{H} \beta$-decay experiments and astrophysical observations) imply that neutrino masses are much smaller than the masses of the charged leptons and quarks. If we take as an indicative upper limit $m_{j} \lesssim 0.5 \mathrm{eV}$, we have $m_{j} / m_{l, q} \lesssim 10^{-6}, l=e, \mu, \tau, q=d, s, b, u, c, t$. It is natural to suppose that the remarkable smallness of neutrino masses is related to the existence of new fundamental mass scale in particle physics, and thus to new physics beyond that predicted by the Standard Model.

A natural explanation of the smallness of neutrino masses is provided by the see-saw mechanism of neutrino mass generation [17]. An integral part of the simplest version of this mechanism - the so-called "type I see-saw", are the $S U(2)_{L}$ singlet $\mathrm{RH}$ neutrinos $\nu_{l R}(\mathrm{RH}$ neutrino fields $\left.\nu_{l R}(x)\right)$. Within the see-saw framework, the latter are assumed to possess a Majorana mass term as well as Yukawa type coupling with the Standard Model lepton and Higgs doublets $\psi_{l L}(x)$ and $\Phi(x)$, respectively, $l=e, \mu, \tau$. In the basis in which the Majorana mass matrix of $\mathrm{RH}$ neutrinos is diagonal, the Majorana mass term of the $\mathrm{RH}$ neutrinos has the standard form $(1 / 2) M_{k} \overline{N_{k}}(x) N_{k}(x), N_{k}(x)$ being the heavy Majorana neutrino field possessing a mass $M_{k}>0$. The fields $N_{k}(x)$ satisfy the Majorana condition $C{\overline{N_{k}}}^{T}(x)=\rho_{k} N_{k}(x)$, where $C$ is the charge conjugation matrix and $\rho_{k}$ is a phase. When the electroweak symmetry is broken spontaneously, the neutrino Yukawa coupling generates a Dirac mass term: $m_{l i}^{D} \overline{\nu_{l L}} N_{i R}(x)+$ h.c., with $m^{D}=v \lambda, \lambda_{l i}$ being the matrix of neutrino Yukawa couplings and $v=174 \mathrm{GeV}$ being the Higgs doublet v.e.v. In the case when the elements of $m^{D}$ are much smaller than $M_{k},\left|m_{l i}^{D}\right| \ll M_{k}, i, k=1,2,3, l=e, \mu, \tau$, the interplay between the Dirac mass term and the Majorana mass term of the heavy Majorana neutrinos $N_{k}$ generates an effective Majorana mass (term) for the LH flavour neutrinos (see, e.g. [17, [18]): $\left(m_{\nu}\right)_{l^{\prime} l} \cong-m_{l^{\prime} j}^{D} M_{j}^{-1}\left(m^{D}\right)_{j l}^{T}$. In grand unified theories, $m^{D}$ is typically of the order of the charged fermion masses. In $S O(10)$ theories, for instance, $m^{D}$ coincides with the up-quark mass matrix. Taking indicatively $m_{\nu} \sim 0.05 \mathrm{eV}, m^{D} \sim 100 \mathrm{GeV}$, one finds $M \sim 2 \times 10^{14} \mathrm{GeV}$, which is close to the scale of unification of the electroweak and strong interactions, $M_{G U T} \cong 2 \times 10^{16} \mathrm{GeV}$. In GUT theories with RH neutrinos one finds that indeed the heavy Majorana neutrinos $N_{j}$ naturally obtain masses which are by few to several orders of magnitude smaller than $M_{G U T}$.

One can similarly obtain an effective Majorana mass term for the LH flavour neutrinos by introducing i) an $S U(2)_{L}$ triplet of leptons, which includes a heavy neutral lepton and has an $S U(2)_{L} \times U(1)_{Y}$ invariant Yukawa coupling with the Standard Model Higgs doublet $\Phi(x)$ and the lepton doublets $\psi_{l L}(x)$ ("type III see-saw mechanism") [19], or ii) by introducing additional neutral $S U(2)_{L}$ singlet fields $S_{\beta L}(x)$ which possess a Majorana mass term and couple to the RH singlet neutrino fields $\nu_{l R}$ ("inverse see-saw scenario") [20].

The estimate of $M_{j}$ given earlier is effectively based on the assumption that the neutrino Yukawa couplings are large: $\left|\lambda_{l i}\right| \sim 1$. The alternative possibility is to have heavy Majorana 
neutrino masses $M_{j}$ in the range of $\sim(100-1000) \mathrm{GeV}$, i.e. TeV scale see-saw generation of neutrino masses. This possibility has received much attention recently (see, e.g. [21]). One of the attractive features of the TeV scale see-saw scenarios is that the heavy Majorana neutrinos $N_{j}$ in such scenarios have relatively low masses which makes $N_{j}$ accessible in the experiments at LHC. This opens up the attractive prospect of having a see-saw mechanism of neutrino mass generation which can be tested experimentally.

One of the characteristic predictions of the type I, type III and the inverse see-saw models is that both the light massive neutrinos and the heavy neutral neutrinos, which play a crucial role in these mechanisms, are Majorana particles. The Majorana nature of the light neutrinos can be revealed in the neutrinoless double beta $\left((\beta \beta)_{0 \nu^{-}}\right)$decay experiments (see, e.g. [18, 22, 23]). As was discussed by a large number of authors (see, e.g. [24] and the references quoted therein), the Majorana nature of the heavy neutrinos of the TeV scale seesaw mechanisms can be established, in principle, in experiments at high energy accelerators, notably at LHC.

In the present article we revisit the low-energy neutrino physics constraints on the $\mathrm{TeV}$ scale type I and inverse see-saw models of neutrino mass generation. We concentrate on the constraints on the parameters of these models which are associated with the non-conservation of the total lepton charge $L$ and thus are directly related with the presence of light and heavy Majorana neutrinos in the indicated models. We discuss the possibility to test the Majorana nature of the heavy Majorana neutrinos, which are an integral part on the indicated mechanisms of neutrino mass generation, at high energy accelerators, and in particular at LHC.

\section{See-Saw Scenarios with Two Mass Scales $\left(M_{D}, M_{R}\right)$}

We consider, first, the standard type I see-saw scenario [17], in which we extend the Standard Model (SM) by adding $k$ "heavy" right-handed $(\mathrm{RH})$ neutrino fields $\nu_{a R}, a=$ $1, \ldots, k, k \geq 2$. We assume that the fields $\nu_{a R}$ are singlets with respect to the Standard Model gauge symmetry group, that they have Yukawa couplings with the left-handed (LH) lepton doublet fields and, in the spirit of the see-saw scenario, possess a "large" Majorana mass. The neutrino mass term in the Lagrangian of the considered extension of the SM is given by:

$$
\mathcal{L}_{\nu}=-\overline{\nu_{\ell L}}\left(M_{D}\right)_{\ell a} \nu_{a R}-\frac{1}{2} \overline{\nu_{a L}^{C}}\left(M_{N}\right)_{a b} \nu_{b R}+\text { h.c. },
$$

where $\nu_{a L}^{C} \equiv C{\overline{\nu_{a R}}}^{T}, C$ being the charge conjugation matrix, $M_{N}=\left(M_{N}\right)^{T}$ is the $k \times k$ Majorana mass matrix of the $\mathrm{RH}$ neutrinos, and $M_{D}$ is a $3 \times k$ neutrino Dirac mass matrix which is generated by the matrix of neutrino Yukawa couplings after the electroweak (EW) symmetry breaking. The matrices $M_{N}$ and $M_{D}$ are complex, in general. The full neutrino mass matrix in eq. (2) can be set in a block diagonal form by the following transformation:

$$
\Omega^{T}\left(\begin{array}{cc}
\mathrm{O} & M_{D} \\
M_{D}^{T} & M_{N}
\end{array}\right) \Omega=\left(\begin{array}{cc}
U^{*} \hat{m} U^{\dagger} & \mathbf{O} \\
\mathbf{O}^{T} & V^{*} \hat{M} V^{\dagger}
\end{array}\right)
$$

where $\Omega$ is a $(3+k) \times(3+k)$ unitary matrix, $\hat{m} \equiv \operatorname{diag}\left(m_{1}, m_{2}, m_{3}\right)$ is a diagonal matrix with the masses of the light Majorana neutrinos, $\hat{M} \equiv \operatorname{diag}\left(M_{1}, M_{2}, \ldots, M_{k}\right)$ is a diagonal matrix 
containing the masses $M_{j}$ of the heavy Majorana neutrino mass eigenstates $N_{j}$ 2. The matrix $\mathbf{O}$ on the left-hand side of (3) is a $3 \times 3$ matrix with all elements equal to zero. The same symbol is used on the right-hand side of (3) to indicate a $3 \times k$ matrix with all null entries. The dimensions of the matrices $\mathbf{O}$ that appear in the block mass matrix decompositions further in the text will not be specified, but can similarly be easily deduced.

The unitary diagonalization matrix $\Omega$ can be formally expressed as the exponential of an antihermitian matrix:

$$
\Omega=\exp \left(\begin{array}{cc}
\mathbf{O} & R \\
-R^{\dagger} & \mathbf{O}
\end{array}\right)=\left(\begin{array}{cc}
\mathbf{1}-\frac{1}{2} R R^{\dagger} & R \\
-R^{\dagger} & \mathbf{1}-\frac{1}{2} R^{\dagger} R
\end{array}\right)+\mathcal{O}\left(R^{3}\right),
$$

where $R$ is a $3 \times k$ complex matrix and the second equality is obtained assuming that $R$ is "small". This assumption will be justified below. In the case under discussion the PMNS [13, 14] neutrino mixing matrix is given by :

$$
U_{\mathrm{PMNS}}=U_{\ell}^{\dagger}(\mathbf{1}+\eta) U
$$

where

$$
\eta=-\frac{1}{2} R R^{\dagger}
$$

and $U$ and $U_{\ell}$ diagonalise the Majorana mass matrix $m_{\nu}$ of the LH flavour neutrinos and the charged lepton mass matrix $m_{\ell}$, respectively:

$$
\begin{gathered}
U^{T} m_{\nu} U=\operatorname{diag}\left(m_{1}, m_{2}, m_{3}\right) \\
U_{\ell} m_{\ell} m_{\ell}^{\dagger} U_{\ell}^{\dagger}=\operatorname{diag}\left(m_{e}^{2}, m_{\mu}^{2}, m_{\tau}^{2}\right)
\end{gathered}
$$

$m_{e}, m_{\mu}$ and $m_{\tau}$ being the charged lepton masses. The matrix $\eta$ parametrises the deviation from unitarity of the neutrino mixing matrix (5) .

In what follows we will work in the basis in which the charged lepton mass matrix is diagonal 3. Accordingly, we set $U_{\ell}=1$ in eq. (5). The charged current (CC) and the neutral current (NC) weak interaction couplings involving the light Majorana neutrinos $\chi_{j}$ with definite mass $m_{j}$ have the form:

$$
\begin{aligned}
\mathcal{L}_{C C}^{\nu} & =-\frac{g}{\sqrt{2}} \bar{\ell} \gamma_{\alpha} \nu_{\ell L} W^{\alpha}+\text { h.c. }=-\frac{g}{\sqrt{2}} \bar{\ell} \gamma_{\alpha}((1+\eta) U)_{\ell i} \chi_{i L} W^{\alpha}+\text { h.c. }, \\
\mathcal{L}_{N C}^{\nu} & =-\frac{g}{2 c_{w}} \overline{\nu_{\ell L}} \gamma_{\alpha} \nu_{\ell L} Z^{\alpha}=-\frac{g}{2 c_{w}} \overline{\chi_{i L}} \gamma_{\alpha}\left(U^{\dagger}\left(1+\eta+\eta^{\dagger}\right) U\right)_{i j} \chi_{j L} Z^{\alpha} .
\end{aligned}
$$

The charged current and the neutral current interactions of the heavy Majorana fields $N_{j}$ with $W^{ \pm}$and $Z^{0}$ read:

$$
\begin{aligned}
\mathcal{L}_{C C}^{N} & =-\frac{g}{2 \sqrt{2}} \bar{\ell} \gamma_{\alpha}(R V)_{\ell k}\left(1-\gamma_{5}\right) N_{k} W^{\alpha}+\text { h.c. }, \\
\mathcal{L}_{N C}^{N} & =-\frac{g}{2 c_{w}} \overline{\nu_{\ell L}} \gamma_{\alpha}(R V)_{\ell k} N_{k L} Z^{\alpha}+\text { h.c. }
\end{aligned}
$$

\footnotetext{
${ }^{2}$ The structure of the neutrino mass matrix shown in (3) appears also in type III see-saw scenario in which the SM is extended by adding $k S U(2)_{L}$ triplet fermion fields.

${ }^{3}$ This can be done without loss of generality.
} 
Therefore, independently of its origin, the mixing of the heavy $(\mathrm{RH})$ Majorana neutrinos with the LH flavour neutrinos is constrained by several low energy data, including $(\beta \beta)_{0 \nu}$-decay [25, 26, 27, 28. More specifically, the diagonal elements of $\eta$ are constrained taking into account the lepton universality tests and the invisible decay width of the $Z^{0}$-boson, while upper bounds on the absolute values of the off-diagonal elements of $\eta$ are obtained from the existing experimental upper limits on the rates of the radiative lepton decays, $\ell_{i} \rightarrow \ell_{j}+\gamma$. For singlet fields $N_{j}$ with masses above the EW symmetry breaking scale, i.e. bigger than $\sim 100 \mathrm{GeV}$, the resulting limits on the non-unitarity of the neutrino mixing matrix read [28, 29]:

$$
|\eta|<\left(\begin{array}{ccc}
4.0 \times 10^{-3} & 1.2 \times 10^{-4} & 3.2 \times 10^{-3} \\
1.2 \times 10^{-4} & 1.6 \times 10^{-3} & 2.1 \times 10^{-3} \\
3.2 \times 10^{-3} & 2.1 \times 10^{-3} & 5.3 \times 10^{-3}
\end{array}\right)
$$

The constraints given above allow to set upper bounds also on the couplings $R V$ of the heavy singlet fields $N_{j}$ with the Standard Model $W^{ \pm}$and charged leptons, and $Z^{0}$ and the LH active neutrinos (see (11) and (12), respectively).

We will standardly assume further that $N_{j}$ have masses $M_{j} \gtrsim 100 \mathrm{GeV}$ and that $M_{N}$ is "much bigger" than $M_{D}$. Using eq. (4) and the expression for the see-saw neutrino mass matrix (3), we obtain the following relations at leading order in $R$ :

$$
\begin{array}{r}
M_{D}-R^{*} M_{N} \simeq \mathbf{O}, \\
-M_{D} R^{\dagger}-R^{*} M_{D}^{T}+R^{*} M_{N} R^{\dagger} \simeq m_{\nu}=U^{*} \hat{m} U^{\dagger}, \\
M_{N}+R^{T} M_{D}+M_{D}^{T} R-V^{*} \hat{M} V^{\dagger} \simeq \mathbf{O} .
\end{array}
$$

Equation (14) implies that under assumptions made the matrix $R$ is indeed "small":

$$
R^{*} \simeq M_{D} M_{N}^{-1}
$$

We can express the light and heavy neutrino mass matrices in (15) and (16) in terms of $M_{N}$ and $R$ :

$$
\begin{aligned}
m_{\nu} & \equiv U^{*} \hat{m} U^{\dagger}=-R^{*} M_{N} R^{\dagger} \\
V^{*} \hat{M} V^{\dagger} & \simeq M_{N}+R^{T} R^{*} M_{N}+M_{N} R^{\dagger} R .
\end{aligned}
$$

The usual type I see-saw expression for the Majorana mass matrix of the LH flavour neutrinos is easily recovered from eqs. (17) and (18): $m_{\nu} \simeq-M_{D} M_{N}^{-1} M_{D}^{T}$.

In the basis we choose to work and up to corrections $\propto R R^{\dagger}$, the elements of the light neutrino mass matrix $m_{\nu}$ are given by: $m_{\nu} \equiv U^{*} \hat{m} U^{\dagger} \cong U_{P M N S}^{*} \hat{m} U_{P M N S}^{\dagger}$. Using the existing upper limits on the absolute scale of neutrino masses and the data on the neutrino mixing angles, obtained in neutrino oscillation experiments, one can derive the ranges of possible values of the elements of $m_{\nu}$ [30]. For the purpose of the present study it is sufficient to use the approximate upper bounds $\left|\left(m_{\nu}\right)_{l^{\prime} l}\right| \lesssim 1 \mathrm{eV}, l, l^{\prime}=e, \mu, \tau$. From eqs. (18) and (19) we obtain to leading order in $R$ :

$$
\sum_{k}\left|(R V)_{l^{\prime} k}^{*} M_{k}(R V)_{k l}^{\dagger}\right| \lesssim 1 \mathrm{eV}, l^{\prime}, l=e, \mu, \tau
$$

In the case of the element $\left(m_{\nu}\right)_{e e}$, the bound follows from the experimental data on the neutrinoless double beta $\left((\beta \beta)_{0 \nu^{-}}\right)$decay [23]. In this case, in addition to the standard 
contribution due to the light Majorana neutrino exchange, the $(\beta \beta)_{0 \nu}$-decay effective Majorana mass $\left(m_{\nu}\right)_{e e}$ (see, e.g. [18, 22]) receives a contribution from the exchange of the heavy Majorana neutrinos $N_{k}$. Taking into account this contribution as well, we get [31, 32, 33]:

$$
\left|\left(m_{\nu}\right)_{e e}\right| \cong\left|\sum_{i}\left(U_{\mathrm{PMNS}}\right)_{e i}^{2} m_{i}-\sum_{k} F\left(A, M_{k}\right)(R V)_{e k}^{2} M_{k}\right|
$$

where $F\left(A, M_{k}\right)$ is a known real (positive) function of the atomic number $A$ of the decaying nucleus and of the mass $M_{k}$ of $N_{k}$ [31, 32, 33]. Using the fact that $U_{P M N S} \hat{m} U_{P M N S}^{T} \cong$ $U \hat{m} U^{T}$ and eqs. (18) and (19), we obtain:

$$
\left|\left(m_{\nu}\right)_{e e}\right| \cong\left|\sum_{k}(R V)_{e k}^{2} M_{k}\left(1+F\left(A, M_{k}\right)\right)\right|
$$

The function $F\left(A, M_{k}\right)$ exhibits a rather weak dependence on $A$, which for the purpose of the present discussion can be neglected, and a relatively strong dependence on $M_{k}$. For $M_{k}=100(1000) \mathrm{GeV}$, an estimate of the largest possible values of $F\left(A, M_{k}\right)$ gives (see, e.g. [34]): $F\left(A, M_{k}\right) \cong 7 \times 10^{-6}\left(7 \times 10^{-8}\right)$. Clearly, in the case of interest the contribution due to the exchange of the heavy Majorana neutrinos in $\left(m_{\nu}\right)_{e e}$ is subdominant and can be neglected. This contribution can be relevant if, for instance, the "leading order" term is very strongly suppressed or if $\sum_{k}(R V)_{e k}^{2} M_{k}=0$.

Using the upper bounds in eq. (20) and barring "accidental" cancellations or extreme fine-tuning (at the level of $\sim 10^{9}$, see, e.g. [36, 37]), we get for the heavy Majorana neutrinos $N_{k}$ having masses $M_{k} \sim M_{R} \geq 100 \mathrm{GeV}$ the well-known strong constraint on the couplings of $N_{k}$ to the weak $W^{ \pm}$and $Z^{0}$ bosons and charged leptons and light neutrinos:

$$
\left|(R V)_{l k}\right| \lesssim 3 \times 10^{-6}\left(\frac{100 \mathrm{GeV}}{M_{R}}\right)^{1 / 2}, \quad l=e, \mu, \tau, j=1,2, \ldots, k .
$$

This constraint 4 makes the heavy Majorana neutrinos $N_{j}$ practically unobservable even at LHC (see, e.g. [24]).

In order for the $\mathrm{CC}$ and $\mathrm{NC}$ couplings of the heavy Majorana neutrinos $N_{j}$ to $W^{ \pm}$and $Z^{0}$, eqs. (11) and (12), to be sufficiently large so that the see-saw mechanism could be partially or completely tested in experiments at the currently operating and planned future accelerators (LHC included), the suppression implied by the inequality (20) should be due to strong mutual compensation between the terms in the sum in the left-hand side of (20). Such cancellations arise naturally from symmetries in the lepton sector, corresponding, e.g. to the conservation of some additive lepton charge $\hat{L}$ (see, e.g. [39, 40, 18, 41, 42]). However, in the exact symmetry limit in this case the heavy neutrinos with definite mass and relatively large couplings to the $W^{ \pm}$and $Z^{0}$ should be Dirac particles, which is possible for all heavy neutrinos only if the number of the RH singlet neutrino fields $k$ is even: $k=2 q, q=1,2, \ldots$ If their number is odd, barring again "accidental" cancellations some (odd number) of the discussed heavy Majorana neutrinos will have strongly suppressed couplings to the $W^{ \pm}$and

\footnotetext{
${ }^{4}$ In principle, one can obtain a more refined constraint on $\left|(R V)_{e k}\right|$ using the existing limits on $\left|\left(m_{\nu}\right)_{e e}\right|$ (see, e.g. 23]). However, the approximate upper bound of $1 \mathrm{eV}$ we are using is sufficient for the purposes of the present discussion.
} 
charged leptons and will be practically unobservable in the current and the future planned accelerator experiments. Further, the spectrum of masses of the three light neutrinos, which depends on the assumed symmetry, typically would not correspond to the observations. The correct light Majorana neutrino mass spectrum can be generated by small perturbations that violate the corresponding symmetry, leading to the non-conservation of the lepton charge $\hat{L}$. These perturbations split each heavy Dirac neutrino into two heavy Majorana neutrinos with close but different masses, i.e. the heavy Dirac neutrinos become heavy pseudo-Dirac neutrinos [43, 44]. The perturbations will have practically negligible effect on the couplings of the heavy Dirac states to the $W^{ \pm}$and $Z^{0}$. If, for instance, $\left|(R V)_{l j}\right| \sim 10^{-3}\left(10^{-4}\right)$, the splitting between the masses of the two heavy Majorana neutrinos forming a pseudo-Dirac pair, as it follows from eq. (20), should satisfy roughly $\left|\Delta M_{P D}\right| \lesssim 1$ (100) MeV for masses of the order of 100 (1000) GeV. Thus, the effect of the perturbations on the low-energy phenomenology of the indicated heavy neutrino states will be essentially negligible and to a high level of precision they will behave like Dirac fermions 5 .

The preceding discussion implies that the Majorana nature of the heavy Majorana neutrinos of the type I see-saw mechanism will be unobservable at LHC and the planned future accelerator experiments. If heavy neutrinos are observed and they are associated with the type I see-saw mechanism without any additional TeV scale "new physics" (e.g. in the form of $Z^{\prime}$ boson associated with an additional $U(1)$ local gauge symmetry, see, e.g. [46] and references quoted therein), they will behave like Dirac fermions to a relatively high level of precision. The observation of effects proving their Majorana nature would imply that these heavy neutral leptons have additional relatively strong non-Standard Model couplings to the Standard Model particles, or that $M_{D} M_{N}^{-1} M_{D}^{T} \cong 0$ and $m_{\nu} \neq 0$ compatible with the observations arises as one and/or two loop higher order correction (see, e.g. [47, 42]).

We will illustrate some of these conclusions/considerations with few simple examples.

The Case of a Broken Symmetry. We will consider first the case when the Majorana mass matrix for the LH flavour neutrinos $m_{\nu} \neq 0$ arises as a result of breaking of a global symmetry corresponding to the conservation of a lepton charge. In the symmetry limit one has $m_{\nu}=0$.

Suppose we have two LH flavour neutrino fields $\nu_{l L}, l=e, \mu$, and two RH neutrino fields $\nu_{a R}, a=1,2$. Let us assign a lepton charge $L_{a}$ to each of the two RH neutrino fields: $L_{a}\left(\nu_{b R}\right)=-\delta_{a b}$, i.e. $\nu_{1 R}$ has lepton charge $L_{1}=-1$ and lepton charges $L_{2}=L_{l}=0, l=e, \mu$. Suppose that the Majorana mass matrix $M_{N}$ in eq. (2) has the form:

$$
M_{N}=\left(\begin{array}{cc}
0 & M_{12} \\
M_{12} & 0
\end{array}\right) .
$$

We take (for concreteness) $M_{12}$ to be real and positive, $M_{12}>0$, and assume also that $M_{12} \geq 100 \mathrm{GeV}$. The motivation for the choice made of the form of $M_{N}$ is that, as is well known, the matrix in eq. (24) has two eigenvalues which have equal absolute values but opposite signs. Thus, one can expect that this may lead to the requisite suppression of the sum in the left-hand side of eq. (20).

The Majorana mass term of the RH neutrinos in eq. (2) with the mass matrix $M_{N}$ given by eq. (24) conserves the lepton charge $\left(L_{1}-L_{2}\right)$. It is diagonalised with the help of a $2 \times 2$

\footnotetext{
${ }^{5}$ For the signatures of production of such TeV scale pseudo-Dirac neutrinos at LHC see, e.g. [24, 45].
} 
orthogonal matrix $\mathbf{V}(\theta)$ with $\theta=\pi / 4$. The heavy Majorana mass-eigenstates $N_{1,2}$ have masses $M_{1}=M_{2}=M_{12} \equiv M$ and satisfy the Majorana conditions $C{\overline{N_{k}}}^{T}=\rho_{k} N_{k}, k=1,2$, where $\rho_{1}=-1$ and $\rho_{2}=+1$ 6. They are equivalent to one heavy Dirac neutrino field $N_{D}=\left(N_{1}+N_{2}\right) / \sqrt{2}$ having a mass $M$. The relations between the fields $\nu_{a R}, \nu_{a L}^{C}, a=1,2$, and the fields $N_{k L}, N_{k R}, k=1,2$, and $N_{D L}, N_{D R}, C{\overline{N_{D L}}}^{T} \equiv N_{D R}^{C}$ and $C{\overline{N_{D R}}}^{T} \equiv N_{D L}^{C}$ have the form:

$$
\begin{aligned}
& \nu_{1 R}=N_{D R}=\frac{1}{\sqrt{2}}\left(N_{1 R}+N_{2 R}\right), \quad \nu_{2 R}=N_{D R}^{C}=\frac{1}{\sqrt{2}}\left(-N_{1 R}+N_{2 R}\right) \\
& \nu_{1 L}^{C}=N_{D L}^{C}=\frac{1}{\sqrt{2}}\left(-N_{1 L}+N_{2 L}\right), \quad \nu_{2 L}^{C}=N_{D L}=\frac{1}{\sqrt{2}}\left(N_{1 L}+N_{2 L}\right) .
\end{aligned}
$$

Let us denote the elements of the Dirac mass matrix $M_{D}$ (see eq. (21)) as $\left(M_{D}\right)_{l k} \equiv m_{l k}^{D}$, $l=e, \mu, k=1,2$. We will assume for simplicity that $M_{D}$ is a real matrix and that $\left|m_{l k}^{D}\right| \ll$ $M_{12}$. The Majorana mass matrix for the LH flavour neutrinos, generated by the see-saw mechanism, has the form:

$$
\begin{aligned}
m_{\nu} & =-R^{*} M_{N} R^{\dagger} \simeq-M_{D} M_{N}^{-1} M_{D}^{T} \\
& =-\frac{1}{M}\left(\begin{array}{cc}
2 m_{e 1}^{D} m_{e 2}^{D} & m_{e 1}^{D} m_{\mu 2}^{D}+m_{e 2}^{D} m_{\mu 1}^{D} \\
m_{e 1}^{D} m_{\mu 2}^{D}+m_{e 2}^{D} m_{\mu 1}^{D} & 2 m_{\mu 1}^{D} m_{\mu 2}^{D}
\end{array}\right) .
\end{aligned}
$$

The matrix of CC and NC couplings of the heavy Majorana neutrinos $N_{1,2}$ to the SM $W^{ \pm}$ and $Z^{0}$ bosons reads:

$$
R V=\frac{1}{\sqrt{2}} \frac{1}{M}\left(\begin{array}{cc}
m_{e 2}^{D}-m_{e 1}^{D} & m_{e 1}^{D}+m_{e 2}^{D} \\
m_{\mu 2}^{D}-m_{\mu 1}^{D} & m_{\mu 1}^{D}+m_{\mu 2}^{D}
\end{array}\right)
$$

The form of $m_{\nu}$, eq. (28) , reflects the the fact that the contributions due to $N_{1}$ and $N_{2}$ tend to cancel each other: we have, for instance, $\left(m_{\nu}\right)_{e e}=-\left[\left(m_{e 2}^{D}+m_{e 1}^{D}\right)^{2}-\left(m_{e 1}^{D}-m_{e 2}^{D}\right)^{2}\right] /(2 M)$, etc.

With $\left|\left(m_{\nu}\right)_{l^{\prime} l}\right| \lesssim 1 \mathrm{eV}$ and $M \geq 100 \mathrm{GeV}$ we indeed get, in general, $\left|(R V)_{l k}\right| \lesssim 10^{-6}$, $l=e, \mu, k=1,2$. The constraints under discussion on some of the elements of the matrix $(R V)$ (and thus on some of the couplings of $N_{1,2}$ to the $W^{ \pm}$and $Z^{0}$ bosons) can be avoided if some of the elements of the Dirac mass matrix are sufficiently small, so that $\left|\left(m_{\nu}\right)_{l^{\prime} l}\right| \lesssim 1 \mathrm{eV}$ is satisfied, and at the same time not all elements of $(R V)$ are suppressed. This possibility can be realised if, for instance, $m_{e 1}^{D} / M$ and $m_{\mu 1}^{D} / M$ are sufficiently small. We will set them to zero in what follows. In this limit we have $\left|\left(m_{\nu}\right)_{l^{\prime} l}\right|=0$. The couplings $m_{e 2}^{D} / M$ and $m_{\mu 2}^{D} / M$ are not constraint, except by the assumption that $\left|m_{l 2}^{D}\right| / M \ll 1, l=e, \mu$.

If $m_{e 1}^{D} / M=m_{\mu 1}^{D} / M=0$, the heavy Majorana neutrinos $N_{1,2}$ couple to the weak $W^{ \pm}$ and $Z^{0}$ bosons only in the combination $\left(N_{1 L}+N_{2 L}\right) / \sqrt{2}=N_{D L}$, i.e. only through the LH component of the Dirac field $N_{D}$. Moreover, in this case there is a conserved lepton charge $\hat{L}=L_{e}+L_{\mu}+\left(L_{1}-L_{2}\right)$. This implies that [18, 39] the theory contains one heavy Dirac neutrino $N_{D}$ and two massless neutrinos $n_{1,2}$. The massless neutrino fields $n_{1 L}$ and $n_{2 L}$ are the dominant components of the LH flavour neutrino fields $\nu_{l L}, l=e, \mu$, while the $N_{D R}$ and

\footnotetext{
${ }^{6}$ The difference in the sign factors in the Majorana conditions for $N_{1,2}$ which have positive masses, reflects the difference in the signs of the two eigenvalues of the matrix $M_{N}$, eq. (24) (for a more detailed discussion see [18).
} 
$N_{D R}^{C}$ are the dominant components of the two RH neutrino fields, $\nu_{a R}, a=1,2$. For the Majorana mass matrix of the LH flavour neutrinos we have in this approximation: $m_{\nu}=0$.

It should be clear that in the approximation being discussed, there are no physical (observable) effects associated with the fact that $N_{1,2}$ are Majorana particles: $N_{1,2}$ always appear in the interaction Lagrangian in the combination $\left(N_{1 L}+N_{2 L}\right) / \sqrt{2}$ which is equivalent to a Dirac fermion. The probability of having, e.g. same sign dilepton events 7 , which would be a signature of the Majorana nature of $N_{1,2}$, is zero. This is a consequence of the fact that the contributions of $N_{1}$ and $N_{2}$ in the amplitudes of the processes of same sign dilepton production are equal in absolute value, but have opposite signs and cancel completely each other.

Indeed, consider the process of same sign di-muon production in $p-p$ collisions, assuming that one of the muons, say $\mu^{-}$, is produced together with real or virtual $N_{1,2}$, while the second $\mu^{-}$originates from the decay $N_{1,2} \rightarrow W^{+}+\mu^{-}$, with virtual or real $W^{+}$(see, e.g. [24]). The $W^{+}$decays further into, e.g. two hadron jets. If in the general case of $M_{1} \neq M_{2}, M_{1}<M_{2}$, the heavy Majorana neutrino $N_{1}$ is real, the invariant mass of the two jets and the second muon should be equal to the mass of $N_{1}$. Note that $N_{1,2}$ are not directly detected. The observation of this process with the characteristic Breit-Wigner enhancement of the cross section due to the $N_{1}$ propagator when the invariant mass of the two jets and the second muon approaches the mass of $N_{1}$, would be a signature of the Majorana nature of $N_{1}$. The part of the amplitude of the process under discussion, which is of interest for the purposes of the present analysis, has the form:

$$
P_{1,2}=\frac{\left(m_{\mu 2}^{D}\right)^{2}}{M_{12}^{2}}\left[\frac{s^{2} M_{2}}{p^{2}-M_{2}^{2}+i \Gamma_{2} M_{2}}-\frac{c^{2} M_{1}}{p^{2}-M_{1}^{2}+i \Gamma_{1} M_{1}}\right]
$$

where $p$ is the four momentum of the real (or virtual) $N_{1,2}, \Gamma_{1,2}$ is the width of $N_{1,2}, c^{2}=$ $\cos ^{2} \theta, s^{2}=\sin ^{2} \theta$, where $\theta$ is the angle in $V$ which diagonalises the RH neutrino mass matrix. In deriving eq. (30) we have taken into account the fact that the sign factors in the Majorana conditions for $N_{1}$ and $N_{2}$ are opposite: $\rho_{1}=-1$ and $\rho_{2}=+1$. For the width of $N_{1,2}$ one has for the ranges of masses of $N_{1,2}$ of interest [24]: $\Gamma_{1(2)} \propto G_{F} M_{1(2)}^{3} \ll M_{1(2)}$. We note that $p^{2}$ is equal to the square of the invariant mass of the two jets and the second muon in the final state of the process (see, e.g. [24]). The above expression is valid for any value of the invariant mass $p^{2}$. It should be emphasised that even when $N_{1}$ is on mass shell, $p^{2}=M_{1}^{2}$, and the second term in eq. (30) dominates due to the fact the $M_{2}$ is significantly bigger than $M_{1}$, the contribution of the virtual $N_{2}$ (i.e., the first term in eq. (30)) is always present in the amplitude. In the specific case we are considering one has actually $M_{1}=M_{2}, \Gamma_{1}=\Gamma_{2}$, $c^{2}=s^{2}=1 / 2$, and therefore $P_{1,2}=0$, as was suggested earlier.

Consider next the "perturbation" of the scheme discussed by having, e.g. $m_{\mu 1}^{D}=0$ but $m_{e 1}^{D} \neq 0$. In this case $\hat{L}$ is no longer conserved: there is no conserved lepton charge in the theory. Correspondingly, $m_{\nu} \neq 0$ (except for the element $\left(m_{\nu}\right)_{\mu \mu}=0$ ). The two heavy Majorana neutrinos $N_{1,2}$ have slightly different masses now, $\left|M_{1}-M_{2}\right| \cong 2\left|m_{e 1}^{D} m_{e 2}^{D}\right| / M$, forming a pseudo-Dirac neutrino $N_{P D}=\left(N_{1}+N_{2}\right) / \sqrt{2}$. [43, 44]. The light neutrinos $n_{1,2}$ have nonzero masses $m_{1,2} \cong m_{e 1}^{D}\left[\sqrt{\left(m_{e 2}^{D}\right)^{2}+\left(m_{\mu 2}^{D}\right)^{2}} \mp m_{e 2}^{D}\right] / M$.

\footnotetext{
${ }^{7}$ One of the charged leptons (say $\mu^{-}$) is produced together with $N_{1,2}$, while the second $\left(\mu^{-}\right)$is supposed to originate from the $N_{1,2}$ decay into $W^{ \pm}+$charged lepton $\left(W^{+}+\mu^{-}\right)$.
} 
If we assume that $\left|m_{e 1}^{D}\right|$ is of the same order as $\left|m_{e 2}^{D}\right|$ and $\left|m_{\mu 2}^{D}\right|$, the constraint (201) applies and $N_{1,2}$ would be hardly observable, e.g. at LHC. If, however, $m_{e 1}^{D} \neq 0$ is generated as a small perturbation, i.e. if $\left|m_{e 1}^{D}\right| \ll\left|m_{e 2}^{D}\right|,\left|m_{\mu 2}^{D}\right|$, one can have $\left(m_{\nu}\right)_{l^{\prime} l} \lesssim 1 \mathrm{eV}$ for relatively large $\left|m_{e 2}^{D}\right| / M$ and/or $\left|m_{\mu 2}^{D}\right| / M$ couplings of $N_{1,2}$ to the $W^{ \pm}$and $Z^{0}$ bosons. This would make possible the production of $N_{1,2}$ with observable rates at LHC. However, also in this case $N_{1}$ and $N_{2}$ couple to $W^{ \pm}$and $Z^{0}$ only in the combination $\left(N_{1 L}+N_{2 L}\right) / \sqrt{2}$. Moreover, they form a pseudo-Dirac neutrino with an extremely small mass splitting. Indeed, if for instance, $\left|m_{e 2}^{D}\right|,\left|m_{\mu 2}^{D}\right| \cong 10^{-3} M$ and $M=100 \mathrm{GeV}$, using $\left|m_{1,2}\right| \lesssim 1 \mathrm{eV}$ we get $\left|m_{e 1}^{D}\right| \lesssim 1 \mathrm{keV}$ and $\left|M_{1}-M_{2}\right| \lesssim 1 \mathrm{eV}$. Actually, we have $\left|M_{1}-M_{2}\right| \cong\left|m_{2}-m_{1}\right|$. Thus, $N_{1}$ and $N_{2}$ form a pseudo-Dirac neutrino which, given the tiny mass splitting between $N_{1}$ and $N_{2}$, will behave for all practical purposes as a Dirac neutrino. The magnitude of all effects revealing the Majorana nature of the heavy neutral leptons $N_{1,2}$ is proportional to their mass difference, i.e. to the factor $\left|m_{e 1}^{D} m_{e 2}^{D}\right| / M^{2}$, which renders these effects unobservable (e.g. at LHC).

We will extend next the previous rather straightforward analysis to the case of $3 \mathrm{LH}$ flavour neutrinos and three $\mathrm{RH}$ neutrinos $\nu_{a R}, a=1,2,3$. Consider for simplicity a model in which one of the three light Majorana neutrinos is massless:

$$
M_{D}=\left(\begin{array}{ccc}
0 & m_{e 2}^{D} & m_{e 3}^{D} \\
0 & m_{\mu 2}^{D} & m_{\mu 3}^{D} \\
0 & m_{\tau 2}^{D} & m_{\tau 3}^{D}
\end{array}\right), \quad M_{N}=\left(\begin{array}{ccc}
M_{11} & 0 & 0 \\
0 & 0 & M_{23} \\
0 & M_{23} & 0
\end{array}\right)
$$

As a consequence of the simplifying choice made $m_{l 1}^{D}=0, l=e, \mu, \tau$, the field $N_{1}=$ $\left(\nu_{1 R}+\nu_{1 L}^{C}\right) / \sqrt{2}$ is decoupled. In the limit $m_{\ell 2}^{D}=0, \ell=e, \mu, \tau$, there is a conserved lepton charge: $L^{\prime} \equiv L_{e}+L_{\mu}+L_{\tau}+L_{3}-L_{2}$. In this case the theory contains three massless and one massive Dirac neutrinos [39] (see also [18]). The three massless neutrinos are the dominant components of the three LH flavour neutrinos. The massive Dirac neutrino $N_{D}=$ $\left(N_{2}+N_{3}\right) / \sqrt{2}$ has a mass $M=M_{23}>0$, where the two heavy Majorana neutrinos $N_{2}$ and $N_{3}$ have the same mass $M_{2}=M_{3}=M_{23}$ and satisfy the Majorana conditions $C{\overline{N_{k}}}^{T}=\rho_{k} N_{k}$, $k=2,3$, where $\rho_{2}=-1$ and $\rho_{3}=+1$. It should be clear that in this case there are no observable effects associated with the Majorana nature of the $N_{2}$ and $N_{3}$.

Consider next the case of $m_{\ell 2,3}^{D} \neq 0, \ell=e, \mu, \tau$. Now there is no conserved lepton charge and the resulting light neutrino mass spectrum has the form:

$$
m_{1}=0, \quad m_{2} \cong \frac{1}{M_{23}}(\sqrt{\Delta}-A), \quad m_{3} \cong \frac{1}{M_{23}}(\sqrt{\Delta}+A),
$$

where

$$
\Delta=\left(m_{e 2}^{D 2}+m_{\mu 2}^{D 2}+m_{\tau 2}^{D 2}\right)\left(m_{e 3}^{D 2}+m_{\mu 3}^{D 2}+m_{\tau 3}^{D 2}\right)
$$

and

$$
A=m_{e 2}^{D} m_{e 3}^{D}+m_{\mu 2}^{D} m_{\mu 3}^{D}+m_{\tau 2}^{D} m_{\tau 3}^{D} .
$$

The heavy neutrino mass spectrum is given by:

$$
M_{1}=M_{11} \quad M_{2} \cong M_{23}-\frac{A}{M_{23}}, \quad M_{3} \cong M_{23}+\frac{A}{M_{23}},
$$

with $\rho_{3}=-\rho_{2}=1$. Note that $M_{3}-M_{2}=2 A / M_{23}=m_{3}-m_{2}$ and therefore, as in the preceding case, the splitting between $M_{3}$ and $M_{2}$ is exceedingly small and unobservable 
in practice. The corrections to the matrix $V$ which diagonalises $M_{N}$ are of the order of $A / M_{23}^{2}$ and are negligible. The elements of the matrix $R$, which parametrises the mixing between the light and the heavy neutrinos, have the form : $R_{\ell 1}^{*}=0, R_{\ell 2}^{*}=m_{\ell 3}^{D} / M_{23}$ and $R_{\ell 3}^{*}=m_{\ell 2}^{D} / M_{23}$, for $\ell=e, \mu, \tau$. For the Majorana mass matrix for the LH flavour neutrinos we get an expression similar to the one in eq. (28): $\left(m_{\nu}\right)_{l l^{\prime}} \cong-\left(m_{l 2}^{D} m_{l^{\prime} 3}^{D}+m_{l 3}^{D} m_{l^{\prime} 2}^{D}\right) / M_{23}$. If we assume that $\left|\left(m_{\nu}\right)_{l l^{\prime}}\right| \lesssim 1 \mathrm{eV}$ and and that $M_{23} \approx 100 \mathrm{GeV}$, we obtain the following constraint on the elements of the Dirac mass term:

$$
\left|m_{\ell 2}^{D} m_{\ell^{\prime} 3}^{D}\right| \lesssim 10^{-7} \mathrm{GeV}^{2}\left(\frac{M_{23}}{100 \mathrm{GeV}}\right), \quad l, l^{\prime}=e, \mu, \tau .
$$

There are two distinct possibilities.

Democratic case: $\left|m_{\ell 2}^{D}\right|$ and $\left|m_{\ell 3}^{D}\right|$ are of the same order. We have:

$$
\left|m_{\ell 2}^{D}\right| \approx\left|m_{\ell 3}^{D}\right| \lesssim 3 \times 10^{-4} \mathrm{GeV}
$$

For $M_{23} \approx 100 \mathrm{GeV}$, this case corresponds to exceedingly small couplings of the heavy Majorana neutrinos $N_{2,3}$ to the $W^{ \pm}$and $Z^{0}:\left|R_{\ell 2,3}\right| \lesssim 3 \times 10^{-6}$. As a consequence, $N_{2,3}$ will be unobservable at LHC.

Hierarchical case: suppose that $\left|m_{\ell 2}^{D}\right| \ll\left|m_{\ell 3}^{D}\right|$. Consider, for instance the possibility:

$$
m_{\ell 2}^{D} \approx 5 \times 10^{-9} \mathrm{GeV}, \quad m_{\ell 3}^{D} \approx 1 \mathrm{GeV} .
$$

This choice allows to have relatively large $\left|R_{\ell 2}\right|,\left|R_{\ell 2}\right| \approx 10^{-2}$ for $M_{23} \approx 100 \mathrm{GeV}$, and thus relatively large $\left|(R V)_{\ell 2}\right|$ and $\left|(R V)_{\ell 3}\right|$. Thus, in principle, $N_{2}$ and $N_{3}$ can be produced with sufficiently large rates at, e.g. LHC, which might allow to observe them. However, it would be hardly possible to obtain experimental evidences for their Majorana nature. Indeed, one has $(R V)_{\ell 2}=(R V)_{\ell 3}=m_{\ell 3}^{D} /\left(\sqrt{2} M_{23}\right)$. Therefore $N_{2}$ and $N_{3}$ couple to a given charged lepton $l$ (neutrino $\nu_{l}$ ) in the weak charged (neutral) current always in the combination $\left(N_{2}+N_{3}\right) / \sqrt{2}=N_{P D}$. As a consequence, the magnitude of all physical effects associated with the Majorana nature of $N_{2}$ and $N_{3}$ will be determined by the mass difference $M_{3}-M_{2}=m_{3}-m_{2} \lesssim 1 \mathrm{eV}$, which renders these effects unobservable in the experiments investigating the properties of the heavy neutrinos $N_{2,3}$.

No Symmetry but $m_{\nu}=0$ at Leading Order. This case has been analised in detail recently in [42, where also the general conditions for having $M_{D} M_{N}^{-1} M_{D}^{T}=0$ have been derived. We will consider one simple realisation of the indicated possibility. Namely, let us assume that $m_{l 1}^{D}, m_{l 2}^{D}=0$, but $m_{l 3}^{D} \neq 0, l=e, \mu, \tau$, and that the matrix $M_{N}$ has the form

$$
M_{N}=\left(\begin{array}{ccc}
M_{11} & 0 & 0 \\
0 & 0 & M_{23} \\
0 & M_{23} & M_{33}
\end{array}\right)
$$

In this case we have $M_{D} M_{N}^{-1} M_{D}^{T}=0$, and thus to leading order $m_{\nu}=0$. Given the assumed simple structure of $M_{D}$ and $M_{N}$, the heavy Majorana neutrino $N_{1}$ having a mass $M_{11}$ decouples from the rest of the neutrino system, while $N_{2,3}$ couple via $W^{ \pm}$and $Z^{0}$ to the Standard Model particles. For $M_{33} \neq 0$, there does not exist a conserved lepton charge and therefore higher order (one or two loop) contributions (see, e.g. [42]) lead to $m_{\nu} \neq 0$. Thus, 
this scheme does not belong to the class of see-saw scenarios (type I or inverse) which are the main subject of this study. Nevertheless, it is instructive to consider on this simple example the constraints that have to be satisfied, which are specifically related to the presence of the heavy Majorana neutrinos. It should be clear from the preceding discussion that all $|\Delta L|=2$ Majorana type effects should vanish in the limit of $M_{33}=0$.

Since $m_{\nu}=0$ to leading order, the constraint given in eq. (20) is not applicable and the couplings of the two heavy Majorana neutrinos $N_{2,3}$ to the $W^{ \pm}$and $Z^{0}$ bosons can be relatively large. This in turn could lead to sufficiently large $N_{2,3}$ production rates at LHC to make the observation of the two heavy Majorana neutrinos possible. We will show that the effects associated with the Majorana nature of $N_{2,3}$ are always proportional to the difference of the masses of $N_{3}$ and $N_{2}$, i.e. to $M_{33}$. This is not surprising since in the limit of $M_{33}=0$, there is a conserved lepton charge and all observable effects related to the Majorana nature of $N_{2,3}$ disappear.

We will assume for simplicity in what follows that $m_{l 3}^{D}, M_{23}$ and $M_{33}$ are real and that $M_{23}, M_{33}>0$. The diagonalisation of the neutrino mass Lagrangian (which includes the Dirac and Majorana mass matrices $M_{D}$ and $M_{N}$ ) shows that there are three massless masseigenstates $n_{1,2,3}$ and three massive Majorana mass-eigenstates $N_{1,2,3}$ with masses $M_{1}=M_{11}$,

$$
M_{2,3}^{\prime}=\frac{1}{2}\left[\sqrt{M_{33}^{2}+4\left(M_{23}^{2}+\left(m_{e 3}^{D}\right)^{2}+\left(m_{\mu 3}^{D}\right)^{2}+\left(m_{\tau 3}^{D}\right)^{2}\right)} \mp M_{33}\right],
$$

satisfying the Majorana conditions: $C{\overline{N_{k}}}^{T}=\rho_{k} N_{k}, k=2,3$, where $\rho_{2}=-1$ and $\rho_{3}=+1$. In this case the angle $\theta$ of the $2 \times 2$ orthogonal sub-matrix of the $3 \times 3$ matrix $\mathbf{V}(\theta)$ which diagonalises $M_{N}$ is different, in general, from $\pi / 4$ : we have $\cos ^{2} \theta=M_{3} /\left(M_{3}+M_{2}\right), \sin ^{2} \theta=$ $M_{2} /\left(M_{3}+M_{2}\right)$, where $M_{2,3}=\left(\sqrt{M_{33}^{2}+4 M_{23}^{2}} \mp M_{33}\right) / 2$ coincide up to the sign of $M_{2}$ with the 2nd and 3rd eigenvalues of the matrix $M_{N}$. We have $M_{3}-M_{2}=M_{3}^{\prime}-M_{2}^{\prime}=M_{33}$. It is not difficult to find the matrices $R=M_{D} M_{N}^{-1}$ and $\eta=-R^{*} R^{\dagger} / 2: \eta_{l l^{\prime}}=-m_{l 3}^{D} m_{l^{\prime} 3}^{D} /\left(2 M_{23}^{2}\right)$. The existing limits on $\left|\eta_{l^{\prime} l}\right|$ imply $\left|\left(m_{e 3}^{D} / M_{23}\right)^{2}\right| \lesssim 8 \times 10^{-3},\left|\left(m_{\mu 3}^{D} / M_{23}\right)^{2}\right| \lesssim 2.4 \times 10^{-4}$. Given these limits we have to a good approximation $M_{2,3}^{\prime} \cong M_{2,3}$. The matrix $R V$ is given by:

$$
R V=\frac{1}{M_{23}}\left(\begin{array}{ccc}
0 & m_{e 3}^{D} \cos \theta & m_{e 3}^{D} \sin \theta \\
0 & m_{\mu 3}^{D} \cos \theta & m_{\mu 3}^{D} \sin \theta \\
0 & m_{\tau 3}^{D} \cos \theta & m_{\tau 3}^{D} \sin \theta
\end{array}\right)
$$

For $M_{33} \ll 2 M_{23}$, we recover the scheme with a heavy pseudo-Dirac neutrino: $N_{2,3}$ have different but close masses, $2\left(M_{3}^{\prime}-M_{2}^{\prime}\right) /\left(M_{3}^{\prime}+M_{2}^{\prime}\right) \cong M_{33} / \sqrt{M_{23}^{2}+\left(m_{e 2}^{D}\right)^{2}+\left(m_{\mu 2}^{D}\right)^{2}} \ll 1$, $\theta \cong \pi / 4$, and $N_{2,3}$ couple to the $W^{ \pm}$and $Z^{0}$ bosons only in the combination $N_{P D L} \cong$ $\left(N_{2 L}+N_{3 L}\right) / \sqrt{2}$.

Consider the contribution to the $(\beta \beta)_{0 \nu}$-decay effective Majorana mass due to the exchange of the two heavy Majorana neutrinos:

$$
\left|\left(m_{\nu}\right)_{e e}\right| \cong\left|\frac{\left(m_{e 3}^{D}\right)^{2}}{M_{23}^{2}}\left(M_{3} F\left(A, M_{3}\right) \sin ^{2} \theta-M_{2} F\left(A, M_{2}\right) \cos ^{2} \theta\right)\right|
$$

The function $F\left(A, M_{k}\right)$ to a very good approximation can be represented as (see, e.g. 32, 33, 35, 34] $) F\left(A, M_{k}\right) \cong\left(M_{a} / M_{k}\right)^{2} f\left(A, M_{k}\right)$, where $M_{a} \cong 0.9 \mathrm{GeV}$ and $f\left(A, M_{k}\right)$ exhibits a weak dependence on $A$ and very weak dependence on $M_{k}$. For $M_{k} \sim(100-1000) \mathrm{GeV}$ 
of interest, the dependence of $f\left(A, M_{k}\right)$ on $M_{k}$ is so weak [34] that can be safely neglected: $f\left(A, M_{2}\right) \cong f\left(A, M_{3}\right) \equiv f(A)$. Taking this into account we get:

$$
\begin{aligned}
\left|\left(m_{\nu}\right)_{e e}\right| & \cong\left|\frac{\left(m_{e 3}^{D}\right)^{2}}{M_{23}^{2}} \frac{M_{a}^{2}}{M_{2} M_{3}} \frac{M_{3}^{2} f\left(A, M_{2}\right)-M_{2}^{2} f\left(A, M_{3}\right)}{M_{3}+M_{2}}\right| \\
& \cong\left|\frac{\left(m_{e 3}^{D}\right)^{2}}{M_{23}^{2}} f(A) M_{a}^{2} \frac{M_{3}-M_{2}}{M_{2} M_{3}}\right| \cong\left|f(A) \frac{\left(m_{e 3}^{D}\right)^{2}}{M_{23}^{2}} \frac{M_{a}^{2}}{M_{23}^{2}} M_{33}\right|,
\end{aligned}
$$

where we expressed the $\cos ^{2} \theta$ and $\sin ^{2} \theta$ in terms of $M_{2,3}$. For $M_{2,3}$ in the range of interest, $M_{k} \sim(100-1000) \mathrm{GeV}$, and for, e.g. ${ }^{76} \mathrm{Ge},{ }^{82} \mathrm{Se},{ }^{130} \mathrm{Te}$ and ${ }^{136} \mathrm{Xe}$, the function $f(A)$ takes the following values [34 (see also [33]) $f(A) \cong 0.079,0.073,0.085$ and 0.068 , respectively; $f(A)$ has a somewhat smaller value of ${ }^{48} \mathrm{Ca}: f\left({ }^{48} \mathrm{Ca}\right) \cong 0.033$. Given $M_{a}$ and $f(A)$, the existing limits on $\left|\left(m_{\nu}\right)_{e e}\right|$ imply a constraint on $\left(\left(m_{e 3}^{D}\right)^{2} / M_{23}^{2}\right) M_{a} M_{33} / M_{23}^{2}$. If $\left(m_{e 3}^{D}\right)^{2} / M_{23}^{2}$ will be determined from an independent measurement, the constraint on $\left|\left(m_{\nu}\right)_{e e}\right|$ will lead to a constraint on $M_{33} / M_{23}$. Taking, e.g. $\left|\left(m_{\nu}\right)_{e e}\right| \lesssim 1 \mathrm{eV}, f(A)=0.078$ (corresponding to ${ }^{76} \mathrm{Ge}$ ) and the maximal value of $\left(m_{e 3}^{D}\right)^{2} / M_{23}^{2}$ allowed by the data and quoted earlier, $8 \times 10^{-3}$, one finds: $M_{33} \lesssim 1.8 \times 10^{-5} M_{23}\left(M_{23} / M_{a}\right)$. For $M_{23}=100 \mathrm{GeV}$ this implies $M_{33} \lesssim 2 \times 10^{-3} M_{23} \cong$ $0.2 \mathrm{GeV} \ll M_{23}$. Such a small $N_{2}-N_{3}$ mass difference would render the Majorana-type effects associated with $N_{2,3}$ hardly observable. If, however, $\left|\left(m_{e 3}^{D} / M_{23}\right)^{2}\right| \lesssim 1.6 \times 10^{-6}$, we get $M_{33} \lesssim M_{23}$.

Consider next the process of same sign di-muon production in $p-p$ collisions, assuming that one of the muons, say $\mu^{-}$, is produced together with real or virtual $N_{2,3}$ in the decay of a virtual $W^{-}$, while the second $\mu^{-}$originates from the decay $N_{2,3} \rightarrow W^{+}+\mu^{-}$, with virtual or real $W^{+}$which decays further into, e.g. two hadronic jets. The analysis is very similar to the one preceding eq. (30) - one has to replace $N_{1(2)}$ with $N_{2(3)}, M_{12}$ with $M_{23}$, $M_{1(2)}$ with $M_{2(3)}$ and $\Gamma_{1(2)}$ with $\Gamma_{2(3)}$. The relevant part of the amplitude of the process under discussion can be obtained from eq. (30) by replacing $m_{\mu 2}^{D}$ with $m_{\mu 3}^{D}$ and making the changes indicated above:

$$
P_{2,3}=\frac{\left(m_{\mu 3}^{D}\right)^{2}}{M_{23}^{2}}\left[\frac{s^{2} M_{3}}{p^{2}-M_{3}^{2}+i \Gamma_{3} M_{3}}-\frac{c^{2} M_{2}}{p^{2}-M_{2}^{2}+i \Gamma_{2} M_{2}}\right],
$$

where now $p$ is the four momentum of the real (or virtual) $N_{2,3}, c^{2}=\cos ^{2} \theta=M_{3} /\left(M_{3}+M_{2}\right.$ ) and $s^{2}=\sin ^{2} \theta=M_{2} /\left(M_{3}+M_{2}\right)$. Also in this case $p^{2}$ is equal to the square of the invariant mass of the two jets and the second muon in the final state of the process (see, e.g. [24]). We note that even when $N_{2}$ or $N_{3}$ is on mass shell, i.e. $p^{2}=M_{2}^{2}$ or $p^{2}=M_{3}^{2}$, and one of the two terms in eq. (45) dominates, the contribution of the second term (i.e. of the virtual $N_{3}$ or $N_{2}$ ) is always present in the amplitude. The expression in eq. (45) can be cast in the form:

$$
P_{2,3}=\frac{\left(m_{\mu 3}^{D}\right)^{2}}{M_{23}^{2}} \frac{M_{2} M_{3}}{M_{3}+M_{2}} \frac{M_{3}^{2}-M_{2}^{2}-i\left(\Gamma_{3} M_{3}-\Gamma_{2} M_{2}\right)}{\left(p^{2}-M_{3}^{2}+i \Gamma_{3} M_{3}\right)\left(p^{2}-M_{2}^{2}+i \Gamma_{2} M_{2}\right)} .
$$

Taking into account that $M_{2} M_{3}=4 M_{23}^{2},\left(M_{3}-M_{2}\right)=M_{33}$, and that $\Gamma_{2(3)} \propto G_{F} M_{2(3)}^{3}$ [24], it is possible to show that $P_{2,3}$ vanishes in the limit of $M_{3}=M_{2}: P_{2,3} \propto\left(M_{3}-M_{2}\right)=M_{33}$. Thus, if $M_{33} \ll M_{23}$, the amplitude of the process $p+p \rightarrow \mu^{-}+\mu^{-}+2$ jets $+X$, generated by the production and decay of real or virtual $N_{2,3}$, will be strongly suppressed. 
The Extreme Fine-tuning Case. It is well known that the see-saw mechanism is underconstrained, namely there is an infinite set of Dirac neutrino mass matrices leading to the observed neutrino parameters. The most general Dirac neutrino mass matrix that satisfies $m_{\nu}=-M_{D} M_{N}^{-1} M_{D}^{T}$, with $m_{\nu} \simeq U_{\mathrm{PMNS}}^{*} \hat{m} U_{\mathrm{PMNS}}^{\dagger}$ and $M_{N} \simeq V^{*} \hat{M} V^{\dagger}$, can be parametrized as [38]:

$$
M_{D}=i U_{\mathrm{PMNS}}^{*} \sqrt{\hat{m}} \Omega \sqrt{\hat{M}} V^{\dagger}
$$

where $\Omega$ is an arbitrary complex orthogonal matrix. Hence, by choosing conveniently the matrix $\Omega$, it is always possible to find a Dirac neutrino mass matrix with at least one large eigenvalue leading to the observed neutrino parameters, while keeping the right-handed neutrino masses in the range $(100-1000) \mathrm{GeV}$. However, as we will show below, this possibility requires in general a huge tuning of parameters.

Let us consider for simplicity the two-generation case. Then, the $\Omega$-matrix can be decomposed in:

$$
\Omega=\left(\begin{array}{cc}
\cos \hat{\theta} & \sin \hat{\theta} \\
-\sin \hat{\theta} & \cos \hat{\theta}
\end{array}\right)=\frac{e^{i \hat{\theta}}}{2}\left(\begin{array}{cc}
1 & -i \\
i & 1
\end{array}\right)+\frac{e^{-i \hat{\theta}}}{2}\left(\begin{array}{cc}
1 & i \\
-i & 1
\end{array}\right) \equiv \Omega_{+}+\Omega_{-},
$$

where $\hat{\theta}=\omega-i \xi$ is a complex parameter. Accordingly, the Dirac neutrino mass matrix can be decomposed as $M_{D}=M_{D+}+M_{D-}$, in a self-explanatory notation.

Taking for definiteness $\xi>0$, it follows that $M_{D+}\left(M_{D_{-}}\right)$grows (decreases) exponentially with $\xi$. Therefore, for sufficiently large $\xi$ it is possible to compensate the huge suppression in eq. (47) from the tiny observed neutrino masses and the light right-handed neutrino masses. Note however that $M_{D-}$ cannot be neglected, even though it is exponentially suppressed compared to $M_{D+}$, since the naive approximation $M_{D} \simeq M_{D+}$ leads to $m_{\nu}=0$, due to $\Omega_{+} \Omega_{+}^{T}=0$. Therefore, reproducing the correct neutrino parameters requires a large amount of tuning, concretely

$$
\frac{\left(M_{D-}\right)_{i j}}{\left(M_{D+}\right)_{i j}} \sim e^{-2 \xi} \sim \frac{m_{i} M_{j}}{\left(M_{D}\right)_{i j}^{2}} .
$$

For instance, demanding $\left(M_{D}\right)_{i j} \sim \mathcal{O}(1 \mathrm{GeV})$ and $M_{j} \sim \mathcal{O}(100 \mathrm{GeV})$ requires a tuning of one part in $10^{9}$ in order to produce a neutrino mass $m_{i} \sim \mathcal{O}\left(10^{-2} \mathrm{eV}\right)$. The fine-tuning problem of this scenario is exacerbated by the presence of radiative corrections to the see-saw parameters which usually spoil the tuning, unless the radiative corrections to the different parameters are highly correlated in such a way that the tuning is preserved. This possibility is extremely unnatural unless originated by an underlying approximate symmetry, such the one proposed before eq.(24).

Furthermore, such light right-handed neutrinos with such large couplings can induce a rate for $(\beta \beta)_{0 \nu}$-decay in conflict with the experimental constraints. The contribution from the right-handed neutrinos to the $(\beta \beta)_{0 \nu}$-decay is approximated by:

$$
\left|\left(m_{\nu}\right)_{e e}\right| \cong\left|\sum_{k} F\left(A, M_{k}\right)(R V)_{e k}^{2} M_{k}\right|
$$

where in this case

$$
R V=-i U_{P M N S} \sqrt{\hat{m}} \Omega^{*} \sqrt{\hat{M}^{-1}}
$$


Using as before that $F\left(A, M_{k}\right) \cong\left(M_{a} / M_{k}\right)^{2} f\left(A, M_{k}\right)$, where $M_{a} \cong 0.9 \mathrm{GeV}$ and $f\left(A, M_{k}\right)$ has a weak dependence with $M_{k}$, we finally obtain:

$$
\left|\left(m_{\nu}\right)_{e e}\right| \cong\left|\sum_{k}\left(U_{\mathrm{PMNS}} \sqrt{\hat{m}} \Omega^{*}\right)_{e k}^{2} \frac{M_{a}^{2}}{M_{k}^{2}} f\left(A, M_{k}\right)\right|
$$

The dominant contribution to this expression is given by the exponentially-enhanced $\Omega_{+}$ matrix, yielding:

$$
\begin{aligned}
\left|\left(m_{\nu}\right)_{e e}\right| & \cong\left|\frac{e^{2 \xi}}{4} \frac{M_{2}^{2}-M_{1}^{2}}{M_{1}^{2} M_{2}^{2}}\left[\sqrt{m_{1}}\left(U_{\mathrm{PMNS}}\right)_{11}-i \sqrt{m_{2}}\left(U_{\mathrm{PMNS}}\right)_{12}\right]^{2} f(A) M_{a}^{2}\right| \\
& \cong\left|\frac{1}{4} \frac{M_{D}^{2}}{M_{2}^{2}} \frac{M_{a}^{2}}{M_{2}^{2}} f(A)\left(M_{2}-M_{1}\right)\right| \cong 10^{-10}\left(M_{2}-M_{1}\right)
\end{aligned}
$$

for $M_{D} \sim \mathcal{O}(1 \mathrm{GeV})$ and $M_{2} \sim \mathcal{O}(100 \mathrm{GeV})$. Therefore, the non-observation of the $(\beta \beta)_{0 \nu^{-}}$ decay requires in this scenario a degeneracy in the right-handed neutrino masses of at least one per cent. As discussed above, the cross section for same sign di-muon production in $p-p$ collisions is proportional to the mass difference of the right-handed neutrinos. Thus, even in this extremely fined-tuned scenario, the Majorana nature of the right-handed neutrinos will be difficult to probe at colliders.

\section{Multiple Mass Scale See-Saw Scenarios}

We will consider next versions of the see-saw scenario, in which we allow couplings of the RH neutrinos with other SM singlets that are therefore involved in the mechanism of generation of light neutrino masses. This implies the presence of more than two mass scales in the latter.

\subsection{Scenario 1}

Consider the following mass Lagrangian:

$$
\mathcal{L}_{\mu}=-\overline{\nu_{\ell L}}\left(m_{D}\right)_{\ell a} \nu_{a R}-\overline{S_{\beta L}}\left(M_{R}\right)_{\beta a} \nu_{a R}-\frac{1}{2} \bar{S}_{\beta L}(\mu)_{\beta \beta^{\prime}} S_{\beta^{\prime} R}^{C}+\text { h.c. }
$$

where $S_{\beta^{\prime} R}^{C} \equiv C{\overline{S_{\beta^{\prime} L}}}^{T}$. We have introduced an arbitrary fixed numbers of RH neutrinos $\nu_{a R}$ and left-handed SM gauge singlets $S_{\beta L}$. We comment on their numbers below.

In what follows we assume that the scale of $M_{R}$ is much bigger than the scales of $m_{D}$ and $\mu$. If we assign one unit of the total lepton charge $L$ to $\nu_{\ell L}, \nu_{a R}$ and $S_{\beta L}$, the terms involving the mass matrices $m_{D}$ and $M_{R}$ conserve $L$, while the term with $\mu \neq 0$ changes $L$ by 2 units. Thus, the $\mu$-term breaks explicitly the $U(1)$ symmetry associated with the lepton charge conservation. In the limit of $\mu=0$, there is a conserved lepton charge and the particles with definite mass are either massless or are massive Dirac fermions. Given the number of the LH flavour neutrino fields $\nu_{l L}, n\left(\nu_{L}\right)$, the numbers of massless and massive Dirac states depends [39] (see also [18]) on the number of RH neutrino fields $\nu_{a R}, n\left(\nu_{R}\right)$, and on the number of LH singlets $S_{\beta L}, n\left(S_{L}\right)$. If, for instance, we have $n\left(\nu_{L}\right)=n\left(\nu_{R}\right)=n\left(S_{L}\right)=1$, 
there is one massless and one massive Dirac neutrinos. In the case of $n\left(\nu_{L}\right)=n\left(\nu_{R}\right)=3$ and, e.g. $n\left(S_{L}\right)=1$, we will have 3 massive Dirac states and one massless neutrino. In the general case the numbers of massive Dirac and massless states are given by [39] $N_{D}=$ $\min \left(n_{L}+n\left(S_{L}\right), n\left(\nu_{R}\right)\right)$ and $N_{0}=\left|n_{L}+n\left(S_{L}\right)-n\left(\nu_{R}\right)\right|$, respectively. Thus, if $n\left(S_{L}\right)=n\left(\nu_{R}\right)$, the number of massless states coincides with the number of the LH flavour neutrinos. This is the case we will be interested in what follows. In this case the three massless states acquire nonzero Majorana masses when $\mu \neq 0$. At the same time each massive Dirac neutrino is split into two Majorana neutrinos having different but very close masses.

In view of the above one can expect that the light neutrino Majorana mass matrix depends linearly on $\mu$ in such a way that in the limit $\mu \rightarrow 0$, the lepton charge conservation symmetry is restored and the three LH flavour neutrinos become massless. The heavy neutrino sector is given by the mixing of the fields $\nu_{a R}$ and $S_{\beta L}$, with relatively small mass splittings. This scenario is the well known inverse see-saw model [20, 40].

Formally, we can derive the expressions of the light neutrino mass matrix $m_{\nu}$ and the corresponding non unitarity mixing parameters $\eta$ from expressions (17), (18) and (6) , by replacing the matrices $M_{D}$ and $M_{R}$ with 8

$$
M_{D} \equiv\left(\begin{array}{cc}
m_{D} & \mathrm{O}
\end{array}\right) \quad M_{N} \equiv\left(\begin{array}{cc}
\mathrm{O} & M_{R}^{T} \\
M_{R} & \mu
\end{array}\right)
$$

We assume further that $M_{R} \gg m_{D}>\mu$. We note that the parameters in the $\mu$ term in the lagrangian (54) can be arbitrarily small because, as we have already noticed before, this is the term in the Lagrangian that breaks explicitly the lepton number. The actual scale of the $\mu$ term depends on the model considered, which at low energy is reduced to an effective field theory described by the Lagrangian (54). This mass scale can, indirectly, affect the non-unitarity effects in the neutrino mixing as well as the couplings of the heavy singlet Majorana fields to the EW gauge bosons, due to the interplay with the other scales in the theory, namely, $m_{D}$ and $m_{R}$ in the see-saw mass formula (see eq. (58)).

The full mass matrix corresponding to eq. (54) takes the form:

$$
\mathcal{M} \equiv\left(\begin{array}{ccc}
\mathrm{O} & m_{D} & \mathrm{O} \\
m_{D}^{T} & \mathrm{O} & M_{R}^{T} \\
\mathrm{O} & M_{R} & \mu
\end{array}\right)
$$

From eq. (17) we obtain:

$$
R^{\dagger}=\left(\begin{array}{c}
-M_{R}^{-1} \mu\left(M_{R}^{-1}\right)^{T} m_{D}^{T} \\
\left(M_{R}^{-1}\right)^{T} m_{D}^{T}
\end{array}\right)
$$

Consequently, light neutrino Majorana mass matrix is given by:

$$
m_{\nu}=U^{*} \hat{m} U^{\dagger} \simeq-R^{*} M_{N} R^{\dagger}=m_{D} M_{R}^{-1} \mu\left(M_{R}^{-1}\right)^{T} m_{D}^{T} .
$$

\footnotetext{
${ }^{8}$ In principle, one can add a non zero $k \times k$ block in the 11 entry of the block Majorana mass matrix $M_{N}$ in eq. (55), which acts as a small perturbation that breaks lepton number explicitly. However, it can be proven that such term does not enter in the expression of the light neutrino mass matrix (see, e.g. 48. for an explicit model) and, therefore, we do not consider this case.
} 
The expression for the non unitary correction matrix $\eta$ includes two terms:

$$
\eta=-\frac{1}{2} m_{D}^{*}\left(M_{R}^{-1}\right)^{*} \mu^{*}\left(M_{R}^{-1}\right)^{\dagger}\left(M_{R}^{-1}\right) \mu\left(M_{R}^{-1}\right)^{T} m_{D}^{T}-\frac{1}{2} m_{D}^{*}\left(M_{R}^{-1}\right)^{\dagger}\left(M_{R}^{-1}\right) m_{D}^{T} .
$$

According to the hierarchy of the mass scales that enter in the theory, the second term is the dominant one and it does not depend on the LNV parameters in $\mu$. Therefore, it is possible to have an observable deviation from unitarity of the PMNS neutrino mixing matrix, without interfering with the tight constraints on the neutrino mass scale, $m_{\nu} \lesssim 1 \mathrm{eV}$, which is proportional to the "small" Majorana mass matrix $\mu$. If we take large non-unitarity effects $\eta \approx 10^{-4}$, and right-handed fields at the scale $M_{R} \approx 1 \mathrm{TeV}$, the lepton number breaking parameters in the lagrangian are given at the scale:

$$
\mu \approx \frac{m_{\nu}}{\eta} \approx 10 \mathrm{keV}
$$

This estimate shows that if the scenario can be tested in neutrino experiments and experiments studying LFV processes (see e.g. [27]), the generation of the $\mu$ term cannot be associated with the EW symmetry breaking. Indeed, assuming new physics at the scale $\Lambda_{N P} \approx 1 \mathrm{TeV}$, a coupling of the form $\left(1 / \Lambda_{N P}\right) H^{\dagger} H \overline{S_{L}} S_{R}^{C}$ (see, e.g. [50]) implies:

$$
\mu \approx \frac{v^{2}}{\Lambda_{N P}} \approx 10 \mathrm{GeV}
$$

It follows from the see-saw mass formula that in this case the non-unitarity effects and the couplings of the heavy neutral fermions to SM particles are respectively $\eta \approx 10^{-11}$ and $R V \approx 10^{-6}$, which are too small to produce measurable effects in the ongoing and the planned future experiments.

Apart from the possibility of observing sizable deviations from unitarity of the light neutrino mixing in the forthcoming experiments, in this scenario the production of the Majorana SM singlets at colliders might not be suppressed. However, all lepton charge violating (LCV) processes involving the heavy singlets, which are associated with their Majorana nature, are strongly suppressed, which renders them unobservable in the current and currently planned future experiments. Therefore, the heavy Majorana singlets, even if produced with sufficiently large rates to be observable, will behave like heavy Dirac neutral singlets to a relatively high level of precision.

In order to illustrate this point, we consider for simplicity the case $n\left(\nu_{L}\right)=2$ and $n\left(\nu_{R}\right)=$ $n\left(S_{L}\right)=1$. We assume all parameters in the theory to be real with positive $M_{R}$ and $\mu$. The Dirac mass term is simply $m_{D}^{T}=\left(\begin{array}{ll}m_{e 1}^{D} & m_{\mu 1}^{D}\end{array}\right)$. Therefore, the non unitary part of the PMNS neutrino mixing matrix reads:

$$
\eta \cong \frac{1}{M_{R}^{2}}\left(\begin{array}{cc}
\left(m_{e 1}^{D}\right)^{2} & m_{e 1}^{D} m_{\mu 1}^{D} \\
m_{e 1}^{D} m_{\mu 1}^{D} & \left(m_{\mu 1}^{D}\right)^{2}
\end{array}\right) .
$$

In the framework considered, the particle content of the theory is given by one massless neutrino, a light Majorana neutrino with mass $m_{\nu}=\mu\left(m_{D} / M_{R}\right)^{2}$ and two heavy Majorana neutrinos $N_{1,2}$ having different but close masses $M_{1} \neq M_{2}$ of the order of $M_{1,2} \cong M_{R} \approx$ $100 \div 1000 \mathrm{GeV}$ and a mass splitting $\left|M_{1}-M_{2}\right| \approx \mu$. The heavy Majorana neutrino fields satisfy the Majorana conditions: $C{\overline{N_{1,2}}}^{T} \equiv \rho_{1,2} N_{1,2}$, with $\rho_{1}=-1$ and $\rho_{2}=1$. In the limit 
$\mu=0$, the lepton charge symmetry is restored and the spectrum consists of two massless LH neutrinos and a Dirac heavy neutrino $N_{D} \equiv\left(-N_{1}+N_{2}\right) / \sqrt{2}$, with $\nu_{R} \equiv N_{D R}$. Therefore, in the LCV regime $(\mu \neq 0)$ we have a heavy pseudo-Dirac neutrino field $N_{P D} \equiv\left(-N_{1}+N_{2}\right) / \sqrt{2}$ which is coupled to the EW gauge bosons via the neutrino mixing. Indeed, the heavy LH components of the two heavy Majorana fields $N_{1}$ and $N_{2}$ have the following couplings to the $W^{ \pm}$and $Z^{0}$ bosons:

$$
R V=\frac{1}{\sqrt{2}}\left(\begin{array}{cc}
-\frac{\mu}{M_{R}} \frac{m_{e 1}^{D}}{M_{R}}-\frac{m_{e 1}^{D}}{M_{R}} & -\frac{\mu}{M_{R}} \frac{m_{e 1}^{D}}{M_{R}}+\frac{m_{e 1}^{D}}{M_{B}} \\
-\frac{\mu}{M_{R}} \frac{m_{\mu 1}^{D}}{M_{R}}-\frac{m_{\mu 1}^{D}}{M_{R}} & -\frac{\mu}{M_{R}} \frac{m_{\mu 1}^{D}}{M_{R}}+\frac{m_{\mu 1}}{M_{R}}
\end{array}\right) .
$$

Consequently, the CC Lagrangian (11) can be cast in the form:

$$
\mathcal{L}_{C C}^{N_{P D}}=-\frac{g}{2 \sqrt{2}}\left(\epsilon_{\ell} \bar{\ell} \gamma_{\alpha}\left(1-\gamma_{5}\right) N_{P D}+\epsilon_{\ell}^{\prime} \overline{N_{P D}} \gamma_{\alpha}\left(1+\gamma_{5}\right) \ell^{C}\right) W^{\alpha}+\text { h.c. },
$$

where $\ell=e, \mu$ and

$$
\epsilon_{\ell}=\frac{m_{\ell 1}^{D}}{M_{R}} \quad \quad \epsilon_{\ell}^{\prime}=\frac{\mu}{M_{R}} \frac{m_{\ell 1}^{D}}{M_{R}} .
$$

Therefore, similarly to the previous scenario, large production rates of the heavy Majorana neutrinos $N_{1,2}$ are possible at colliders, but LCV decays (processes) associated with their Majorana nature are strongly suppressed. Indeed, the suppression factor for the rate of the LCV decay $N_{P D} \rightarrow \ell^{+} W^{-}$is given by: $\left|\epsilon_{\ell}^{\prime}\right|^{2} \cong m_{\nu}^{2} /\left(\eta M_{R}^{2}\right)$, and for $\eta \approx 10^{-4}$ and $M_{R} \approx 100$ $\mathrm{GeV}$ we have $\left|\epsilon_{\ell}^{\prime}\right|^{2} \approx 10^{-18}$.

\subsection{Scenario 2}

We consider now a variation of the previous scenario in which the source of the lepton number breaking parameter is a (small) Dirac-type mass term between the heavy singlets. The Dirac and Majorana neutrino mass matrices of this model are the following:

$$
M_{D} \equiv\left(\begin{array}{cc}
m_{D} & \mu^{\prime}
\end{array}\right) \quad M_{N} \equiv\left(\begin{array}{cc}
\mathrm{O} & M_{R}^{T} \\
M_{R} & \mathrm{O}
\end{array}\right)
$$

We assume also in this case a hierarchical mass pattern: $M_{R} \gg m_{D}, \mu^{\prime}$. The neutrino mass matrix and the deviation of $U_{\text {PMNS }}$ from unitarity in the scheme considered are given by:

$$
\begin{aligned}
U^{*} \hat{m} U^{\dagger} & \simeq-R^{*} M_{N} R^{\dagger}=-m_{D} M_{R}^{-1} \mu^{T}-\mu^{\prime}\left(M_{R}^{-1}\right)^{T} m_{D}^{T} \\
\eta & =-\frac{1}{2} m_{D}^{*}\left(M_{R}^{-1}\right)^{*}\left(M_{R}^{-1}\right)^{T} m_{D}^{T}-\frac{1}{2} \mu^{\prime *}\left(M_{R}^{-1}\right)^{\dagger}\left(M_{R}^{-1}\right) \mu^{\prime T} .
\end{aligned}
$$

In the case considered, a large mixing between the light and heavy singlet neutrinos corresponds to a much smaller lepton number breaking scale $\mu^{\prime}$, which is given roughly by $\mu^{\prime} \approx m_{\nu} / \sqrt{|\eta|} \approx 10 \mathrm{eV}$. It is not difficult to prove that also in this case the lepton number non-conserving couplings of the heavy singlet neutrinos to the EW gauge bosons $W^{ \pm}$and $Z^{0}$ are exceedingly small which makes the $|\Delta L|=2$ effects unobservable: the couplings of interest are given approximately by $m_{\nu} /\left(\sqrt{|\eta|} M_{R}\right)$ and for, e.g. $\eta \approx 10^{-9}$ and $M_{R} \approx 100$ $\mathrm{GeV}$ we have $\left|m_{\nu}\right| /\left(\sqrt{|\eta|} M_{R}\right) \approx 10^{-7}$. 


\section{Avoiding the Constraints: Non-Singlet Heavy Neu- trinos}

The previous general argument shows that the requirement $\left|\left(m_{\nu}\right)_{l l^{\prime}}\right| \lesssim 1 \mathrm{eV}, l, l^{\prime}=e, \mu, \tau$, translates into an extremely suppressed charged and neutral current interactions of the heavy Majorana fields $N_{j}$ with the Standard Model charged leptons and neutrinos, unless the heavy Majorana neutrinos form a pseudo-Dirac pair. Therefore, if these interactions are the only portal to the Standard Model, the Majorana nature of the heavy neutrinos will not be detected in collider experiments: either the production cross section is highly suppressed or the heavy neutrinos behave to a high level of precision as Dirac fermions.

This result may not be valid if there exist additional $\mathrm{TeV}$ scale interaction terms in the Lagrangian between the heavy Majorana neutrinos and the Standard Model particles. If this is the case, the production cross section of heavy neutrinos will not necessarily be suppressed, while their charged and neutral current interactions with the Standard Model charged leptons and neutrinos can still be tiny.

One possibility is the existence of an extra $U(1)$ local gauge symmetry, which is broken at the $\mathrm{TeV}$ scale and under which the Standard Model particles and the heavy $(\mathrm{RH})$ neutrinos are charged (see, e.g. [46]). In this case, the production cross section of two heavy Majorana neutrinos can be largely enhanced. At the same time, the heavy neutrinos can decay only into Standard Model particles and they can do it only through the tiny charged current and neutral current couplings. This implies that the heavy neutrinos will be relatively long-lived and thus will have a relatively large decay length which in turn will yield a characteristic displaced vertex in the detectors (see, e.g. [49]). More importantly, if the heavy neutrinos are true Majorana particles, their production and decay will lead to events with a pair of same-sign muons in the final state. The cross section can be large enough to allow the observation of this lepton number violating process at colliders.

A second example can be found in the TeV scale type III see-saw mechanism. In this case, the heavy states form an $S U(2)_{L}$ triplet of leptons, $L^{ \pm}, L^{0}$, with essentially the same mass, $L^{ \pm}$being somewhat heavier than $L^{0}$ (see, e.g. [49]). A pair of these leptons, say $L^{+}$ and $L^{0}$, can be produced in colliders via their gauge coupling to the $W^{ \pm}$-boson. The charged heavy lepton $L^{+}$can decay into $\mu^{+}+Z^{0}$. The heavy neutral Majorana lepton $L^{0}$ has an interaction Lagrangian with the Standard Model charged leptons and neutrinos, which is similar to that given in eqs. (91) and (10). Thus, being a Majorana particle, $L^{0}$ can decay into $\mu^{+}+W^{-}$, leading to same-sign dimuon (plus 4 jets) events with observable displaced vertices of the two muons in the detectors. Detailed calculations have shown that for masses of $L^{+}$and $L^{0}$ not exceeding $1000 \mathrm{GeV}$, the $\mu^{+} \mu^{+}+4$ jets events can have observable rates at LHC (see, e.g. [49]).

\section{Conclusions}

In this article we have discussed the possibility to test the Majorana nature of the heavy Majorana neutrinos $N_{j}$ which are an integral part of the TeV scale type I and inverse see-saw scenarios of neutrino mass generation. In the indicated $\mathrm{TeV}$ scale see-saw scenarios the heavy Majorana neutrinos typically have masses in the range of $M_{j} \sim(100-1000) \mathrm{GeV}$. The fact that $N_{j}$ are Majorana particles can be revealed by observation of processes with real or virtual 
$N_{j}$, in which the total lepton charge $L$ changes by two units, $|\Delta L|=2$, like $p+p \rightarrow \mu^{-}+\mu^{-}+$ 2 jets $+X$ at LHC, etc. We have shown that the physical effects associated with the Majorana nature of these heavy neutrinos $N_{j}$, are so small that they are unlikely to be observable in the currently operating and future planned accelerator experiments (including LHC). This is a consequence of the existence of very strong constraints on the parameters and couplings, responsible for the corresponding $|\Delta L|=2$ processes in which $N_{j}$ are involved, and/or on the couplings of $N_{j}$ to the weak $W^{ \pm}$and $Z^{0}$ bosons. The constraints are related to the fact that the elements of the Majorana mass matrix of the left-handed flavour neutrinos $m_{\nu}$, generated by one of the indicated see-saw mechanisms, should satisfy, in general, $\left|\left(m_{\nu}\right)_{l l^{\prime}}\right| \lesssim 1 \mathrm{eV}$, $l, l^{\prime}=e, \mu, \tau$; in the case of the $\left(m_{\nu}\right)_{e e}$ element, the upper limit follows from the experimental searches for neutrinoless double beta $\left((\beta \beta)_{0 \nu^{-}}\right)$decay. Even in the case of extreme fine tuning (at the level of one part in $10^{9}$ or $10^{10}$ ), in which the neutrino Yukawa couplings can be of order 1, the upper limit on $\left|\left(m_{\nu}\right)_{e e}\right|$ obtained in the $(\beta \beta)_{0 \nu}$-decay experiments implies a strong constraint on the $|\Delta L|=2$ heavy Majorana neutrino mass splitting(s) (or masses), which makes it very difficult (if not impossible) to probe the Majorana nature of the heavy Majorana neutrinos in experiments at colliders. The simple illustrative examples we have considered suggest that if the heavy Majorana neutrinos $N_{j}$ are observed and they are associated with the type I or inverse see-saw mechanisms and no additional TeV scale "new physics", they will behave like Dirac fermions to a relatively high level of precision, being actually pseudo-Dirac particles. The observation of effects proving the Majorana nature of $N_{j}$ would imply that these heavy neutrinos have additional relatively strong couplings to the Standard Model particles (as, e.g. in the TeV scale type III see-saw scenario), or that the light neutrino masses compatible with the observations are generated by a mechanism other than the see-saw (e.g., radiatively at one or two loop level) in which the heavy singlet Majorana neutrinos $N_{j}$ are nevertheless involved.

The considerations presented in this article and the conclusions reached concern a rather large number of TeV scale see-saw models discussed in the literature (see, e.g. [21, 50]).

\section{Acknowledgments}

We would like to thank S. Pascoli for discussions. S.T.P. whishes to thank M. Blennow, E. Fernandez-Martinez, J. Lopez-Pavon and J. Menendez for useful correspondence. This work was supported in part by the INFN program on "Astroparticle Physics", by the World Premier International Research Center Initiative (WPI Initiative), MEXT, Japan (A.I. and S.T.P.), the EU-RTN Programme (Contract No.MRTN-CT-2006-035482) Flavianet (E.M.) and by the DFG cluster of excellence "Origin and Structure of the Universe" (A.I.).

\section{References}

[1] B.T. Cleveland et al., Astrophys. J. 496, 505 (1998).

[2] Y. Fukuda et al. [Kamiokande Collaboration], Phys. Rev. Lett. 77, 1683 (1996).

[3] J.N. Abdurashitov et al., Phys. Rev. C 80, 015807 (2009).

[4] P. Anselmann et al., Phys. Lett. B 285 (1992) 376; W. Hampel et al., Phys. Lett. B 447 (1999) 127; M. Altmann et al., Phys. Lett. B 616 (2005) 174. 
[5] S. Fukuda et al. [Super-Kamiokande Collaboration], Phys. Lett. B539 (2002) 179.

[6] Q.R. Ahmad et al. [SNO Collaboration], Phys. Rev. Lett. 87 (2001) 071301 and 89 (2002) 011301.

[7] Y. Fukuda et al. [Super-Kamiokande Collaboration], Phys. Rev. Lett. 81 (1998) 1562.

[8] Y. Ashie et al. [Super-Kamiokande Collaboration], Phys. Rev. Lett. 93 (2004) 101801.

[9] K. Eguchi et al. [KamLAND Collaboration], Phys. Rev. Lett. 90 (2003) 021802; T. Araki et al., Phys. Rev. Lett. 94 (2005) 081801.

[10] C. Arpesella et al., Phys. Lett. B 658 (2008) 101; Phys. Rev. Lett. 101 (2008) 091302.

[11] M. H. Ahn et al. [K2K Collaboration], Phys. Rev. D 74 (2006) 072003.

[12] D.G. Michael et al. [MINOS Collaboration], Phys. Rev. Lett. 97 (2006) 191801; P. Adamson et al., Phys. Rev. Lett. 101 (2008) 131802.

[13] B. Pontecorvo, Zh. Eksp. Teor. Fiz. (JETP) 33 (1957) 549 and 34 (1958) 247.

[14] Z. Maki, M. Nakagawa and S. Sakata, Prog. Theor. Phys. 28 (1962) 870.

[15] B. Pontecorvo, Sov. Phys. JETP 26 (1968) 984. [Zh. Eksp. Teor. Fiz. 53, 1717 (1967)].

[16] C. Amsler et al. [Particle Data Group], Phys. Lett. B 667 (2008) 1.

[17] P. Minkowski, Phys. Lett. B 67 (1977) 421; M. Gell-Mann, P. Ramond and R. Slansky, Proceedings of the Supergravity Stony Brook Workshop, New York 1979, eds. P. Van Nieuwenhuizen and D. Freedman; T. Yanagida, Proceedinds of the Workshop on Unified Theories and Baryon Number in theUniverse, Tsukuba, Japan 1979, ed.s A. Sawada and A. Sugamoto; R. N. Mohapatra and G. Senjanovic, Phys. Rev. Lett. 44 (1980) 912.

[18] S. M. Bilenky and S. T. Petcov, Rev. Mod. Phys. 59 (1987) 671.

[19] R. Foot, H. Lew, X. G. He and G. C. Joshi, Z. Phys. C 44 (1989) 441.

[20] R. N. Mohapatra and J. W. F. Valle, Phys. Rev. D 34 (1986) 1642.

[21] J. Chakrabortty, arXiv:1003.3154; C. Wei,arXiv:1003.1468; F. M. L. de Almeida et al., Phys. Rev. D 81 (2010) 053005; H. Zhang and S. Zhou, Phys. Lett. B 685 (2010) 297; P. S. B. Dev and R. N. Mohapatra, Phys. Rev. D 81 (2010) 013001; Z. z. Xing and S. Zhou, Phys. Lett. B 679 (2009) 249.

[22] S.M. Bilenky, S. Pascoli and S.T. Petcov, Phys. Rev. D 64 (2001) 053010; S.T. Petcov, Phys. Scripta T 121 (2005) 94; S. Pascoli and S. T. Petcov, Phys. Rev. D 77 (2008) 113003.

[23] See, e.g. C. Aalseth et al., arXiv:hep-ph/0412300; C.E. Aalseth et al. [IGEX Collaboration], Phys. Atomic Nuclei 63 (2000) 1225; H. V. Klapdor-Kleingrothaus et al., Phys. Lett. B 586 (2004) 198; A. S. Barabash [NEMO Collaboration], arXiv:0807.2336 [nuclex]; C. Arnaboldi et al. [CUORICINO Collaboration], Phys. Rev. C 78 (2008) 035502. 
[24] A. Atre et al., JHEP 0905 (2009) 030.

[25] F. del Aguila, J. A. Aguilar-Saavedra and R. Pittau, J. Phys. Conf. Ser. 53 (2006) 506 arXiv:hep-ph/0606198].

[26] E. Fernandez-Martinez et al., Phys. Lett. B 649 (2007) 427.

[27] A. Abada et al., JHEP 0712 (2007) 061.

[28] S. Antusch, J. P. Baumann and E. Fernandez-Martinez, Nucl. Phys. B 810 (2009) 369.

[29] S. Antusch et al., JHEP 0610 (2006) 084.

[30] A. Merle and W. Rodejohann, Phys. Rev. D 73 (2006) 073012.

[31] A. Halprin, S.T. Petcov and S.P. Rosen, Phys. Lett. B 125 (1983) 335.

[32] J. Vergados, Nucl. Phys. B 218 (1983) 109.

[33] W.C. Haxton and J. Stephenson, Prog. Part. Nucl. Phys. 12 (1984) 409.

[34] M. Blennow et al., arXiv:1005.3240.

[35] H. Paes et al., Phys. Lett. B 498 (2001) 35.

[36] J. Kersten and A. Y. Smirnov, Phys. Rev. D 76 (2007) 073005.

[37] Z. z. Xing, Phys. Lett. B 679 (2009) 255.

[38] J. A. Casas and A. Ibarra, Nucl. Phys. B 618 (2001) 171.

[39] C.N. Leung and S.T. Petcov, Phys. Lett. B 125 (1983) 461.

[40] D. Wyler and L. Wolfenstein, Nucl. Phys. B 218 (1983) 205.

[41] G. C. Branco, W. Grimus and L. Lavoura, Nucl. Phys. B 312 (1989) 492.

[42] R. Adhikari and A. Raychaudhuri, arXiv:1004.5111.

[43] L. Wolfenstein, Nucl. Phys. B 186 (1981) 147.

[44] S.T. Petcov, Phys. Lett. B 110 (1982) 245.

[45] F. del Aguila and J. A. Aguilar-Saavedra, Phys. Lett. B 672 (2009) 158 [arXiv:0809.2096 [hep-ph]].

[46] K. Huitu et al., Phys. Rev. Lett. 101 (2008) 181802.

[47] S.T. Petcov and S. Toshev, Phys. Lett. B 143 (1984) 175; K. Babu and E. Ma, Phys. Rev. Lett. 61 (1988) 674.

[48] E. Dudas and C. A. Savoy, Acta Phys. Polon. B 33 (2002) 2547.

[49] R. Franceschini, T. Hambye and A. Strumia, arXiv:0805.1613.

[50] F. Bonnet, D. Hernandez, T. Ota and W. Winter, JHEP 0910 (2009) 076. 